\title{
ENTRY AND EXIT DECISION PROBLEM WITH IMPLEMENTATION DELAY
}

\author{
MARIUS COSTENIUC, ${ }^{*}$ Swiss Re \\ MICHAELA SCHNETZER,** \\ LUCA TASCHINI, ${ }^{* * *}$ University of Zürich
}

\begin{abstract}
We study investment and disinvestment decisions in situations where there is a time lag $d>0$ from the time $t$ when the decision is taken to the time $t+d$ when the decision is implemented. In this paper we apply the probabilistic approach to the combined entry and exit decisions under the Parisian implementation delay. In particular, we prove the independence between Parisian stopping times and a general Brownian motion with drift stopped at the stopping time. Relying on this result, we solve the constrained maximization problem, obtaining an analytic solution to the optimal 'starting' and 'stopping' levels. We compare our results with the instantaneous entry and exit situation, and show that an increase in the uncertainty of the underlying process hastens the decision to invest or disinvest, extending a result of Bar-Ilan and Strange (1996).
\end{abstract}

Keywords: Brownian excursion; implementation delay; Parisian option; optimal stopping; Wald's identity

2000 Mathematics Subject Classification: Primary 60G40; 60J65; 62L15

\section{Introduction}

Numerous investment and disinvestment decisions are characterized by a significant implementation lag which has a profound impact on the profitability of the economic or financial decisions undertaken. In fact, depending on the evolution of the decision variable during the implementation lag, the investment or disinvestment opportunity may lose part of its attractiveness. Although in the past two decades a number of investment and disinvestment models have been extensively studied, the problem of the existence of a time lag between the decision time and the implementation time has not received much attention in the literature (for exceptions, see Bar-Ilan and Strange (1996) and Gauthier and Morellec (2000), who studied investments with implementation delay, and Øksendal (2005), who studied optimal disinvestment with delayed information). It is our purpose in this paper to analyze the effect of implementation lags on reversible decisions and on simultaneously determined optimal investment and disinvestment levels. Our analysis differs significantly from the 'constructionlag' or 'time-to-build' literature, where the lag refers to the time between the decision to invest and the receipt of the project's first revenues (see Majd and Pindyck (1987) and Pindyck (1991), (1993)). In our case the lag measures a systematic delay that occurs before the investment or

Received 1 July 2008; revision received 29 October 2008.

* Postal address: Swiss Re, Zürich, Switzerland. Email address: marius.costeniuc@ gmail.com

** Email address: michaela.schnetzer@gmail.com

*** Postal address: Swiss Banking Institute, University of Zürich, Plattenstrasse 32, 8032 Zürich, Switzerland.

Email address: taschini@isb.uzh.ch 
disinvestment project effectively takes place, i.e. before the consequence of hitting the trigger price comes into play.

The combined investment and disinvestment decision problem, also called the entry and exit problem, was initially discussed in Brennan and Schwartz (1985) and McDonald and Siegel (1986). Applying the option pricing theory developed in Black and Scholes (1973) and Merton (1973), they evaluated active and inactive firms and defined the concepts of option to enter and option to abandon as part of the firms' value. A rigorous mathematical treatment of the investment and disinvestment decision problem was proposed in Brekke and Øksendal (1994). The authors analyzed the entry-exit decision problem by applying both option pricing and dynamic programming theories. They gave a formal proof of the existence of a solution and extended the classical approach to considering the case of a finite resource. Duckworth and Zervos (2000) proposed a general model for an investment producing a single commodity which relied on the complementarity of the contingent claim approach and the dynamic programming approach. For a rigorous proof of this relationship, we refer the reader to Knudsen et al. (1999). A formal and complete discussion of the entry and exit problem-based on differential equations when the dynamics of the system follow a geometric Brownian motion-was presented in Dixit (1989). Using the notion of reflected backward stochastic differential equations, Hamadène and Jeanblanc (2007) solved the investment/disinvestment problem for a very general underlying process.

Although these papers have taken a great step toward a better understanding of investment and disinvestment decisions, they assumed that the project is brought on line immediately after the decision to invest is made. The same holds true for the disinvestment option. Nevertheless, the complexity of these decisions and the constraints they are subject to are not properly modeled. To model the delay between the decision to invest (or disinvest) and the implementation process, we follow the study in Bar-Ilan and Strange (1996) and consider that an investment (disinvestment) project initiated at a precise date will be implemented only at a specific period of time. Bar-Ilan and Strange (1996) focused on the effects of time lags on irreversible investments and presented analytical solutions to this problem. With an application to portfolio disinvestment and to resource extraction, Øksendal (2005) identified optimal stopping rules by reducing the problem with delayed information to a classical optimal exit problem. Gauthier and Morellec (2000) studied the implementation delay that affects the capital budgeting process (for which see Harris and Raviv (1996)). The paper addressed the issue of an investment decision under the Parisian implementation delay using the probabilistic approach.

The aim of this paper is to study the effects of implementation delay in the simplest possible model of an uncertain, reversible decision process and to present analytic solutions for optimal entry and exit levels. This framework characterizes numerous industrial-production processes. In the industrial-production context, investing indicates the decision to undertake or start a production process, whereas disinvesting indicates the decision to undo or change a specific production process. A typical example, also introduced in Hamadène and Jeanblanc (2007), is the energy-industry sector. Fuel-burning utilities which generate electricity burning either gas or coal have a co-production option. Each facility conveniently adapts its fuel inputs according to the price evolution of these factors on international exchanges. However, each utility needs some time to implement fuel switching. Fuel switching is the extent to which a producer can reduce the use of a certain type of energy — coal, for instance-and uptake another source of energy — gas - in its place. Ideally, the decision to fuel switch takes place when the underlying variable, i.e. the fuel price, hits a prespecified barrier level. In fact, the existence of physical 
and technical constraints allows the implementation of the new production process after only a given time interval (see Tseng and Barz (2002) and Deng and Oren (2003)).

As in Gauthier and Morellec (2000), we apply the probabilistic approach, but we apply it to the combined investment and disinvestment decision under the Parisian implementation delay. The Parisian delay reflects the will of any firm to verify that market conditions remain favorable or unfavorable during the implementation lag of the investment or disinvestment decision, respectively. The Parisian criterion originates from a relatively new type of financial option contract introduced in Chesney et al. (1997) and termed a Parisian option. Such a contract corresponds to a generalization of a barrier-type option. More precisely, a Parisian option gets activated or deactivated when the underlying process has spent a sufficient amount of time above or, respectively, below the barrier level. Literature addressing the mathematical and computational aspects related to this new option contract is extensive (see Schröder (2003), Avellaneda and $\mathrm{Wu}$ (1999), Haber et al. (1999), and the references therein). However, to the authors' knowledge, only Gauthier and Morellec (2000) use the Parisian criterion for the appraisal of investments in a real option context.

The probabilistic approach leads to more tractable valuation results than does the partial differential equation approach. Relying on standard mathematical results, we prove the independence between Parisian stopping times and the underlying process stopped at the stopping time, obtaining a quite remarkable result. More precisely, in Lemma C. 1 we prove that such an independence property holds for a Brownian motion with drift and not just for a standard Brownian motion. This makes our study significantly different from the analysis in Chesney et al. (1997) and in Gauthier and Morellec (2000). An interesting contact with Wald's identity is discussed. We derive a sufficient condition in order to obtain correctly ordered Parisian optimal levels, i.e. with the investment level higher than the disinvestment level. Finally, a numerical exercise is performed, comparing the delayed investment and disinvestment decision problem with the corresponding instantaneous problem. Our results confirm that an increase in uncertainty postpones instantaneous investments (see Pindyck (1991)) and that the value of investments and disinvestments under the Parisian criterion is lower compared to the corresponding instantaneous cases (Gauthier and Morellec (2000) were the first to obtain this result in the investment case). Moreover, extending the results of Bar-Ilan and Strange (1996) to the disinvestment case, we show that an increase in the uncertainty of the underlying process also hastens the decision to disinvest.

The paper is organized as follows. In Section 2 we introduce the model for the investment and disinvestment decision problem in the context of the Parisian stopping times. In Section 3 we solve the constrained maximization problem and derive sufficient conditions to obtain the optimal triggering levels correctly ordered. In Section 4 we conclude with a numerical comparison.

\section{Problem formulation}

Following the literature on real options (see Dixit and Pindyck (1994) and Bar-Ilan and Strange (1996)), we model optimal investment and disinvestment decisions as the valuation of a perpetual American option contract. Agents are risk neutral and the firm has an investment opportunity in a nontraded asset yielding stochastic returns. Markets are incomplete in the sense that it is impossible to buy an asset or a dynamic portfolio of assets spanning the stochastic changes in the value of the project. There is no futures market for the decision variable and the size of the investment project prevents the firm from taking a position on such a market. As mentioned before, the Parisian criterion reflects the will of the firm to check that the market 
conditions remain favorable or unfavorable during the implementation delay of the investment or disinvestment decision, respectively. In this section we give the mathematical formulation of the Parisian criterion, and in the next section we determine the value of investment and disinvestment decisions under the Parisian criterion.

At any time $t$ the firm can invest in a project yielding an operating profit that depends on the instantaneous cash flow $\left(S_{t}, t \geq 0\right)$. We assume that $S_{t}$ follows a geometric Brownian motion,

$$
\frac{\mathrm{d} S_{t}}{S_{t}}=\mu \mathrm{d} t+\sigma Z_{t}, \quad S_{0}=x,
$$

where $\mu$ and $\sigma$ are constants and $\left(Z_{t}, t \geq 0\right)$ is a Brownian motion defined on a filtered probability space $\left(\Omega, \mathcal{F},\left(\mathcal{F}_{t}\right)_{t \geq 0}, \mathrm{P}\right)$. We denote by $V_{t}$ the expected sum of the discounted cash flows from $t$ to $\infty$,

$$
V_{t}=\mathrm{E}_{t}\left[\int_{t}^{\infty} \mathrm{e}^{-\rho(u-t)} S_{u} \mathrm{~d} u\right]
$$

where the discount rate $\rho$ is constant and $\mathrm{E}_{t}[\cdot]$ stands for the conditional expectation $\mathrm{E}\left[\cdot \mid \mathcal{F}_{t}\right]$.

Lemma 2.1. Assume that $S_{t}$ follows a geometric Brownian motion and that $\rho>\mu$. Then $V_{t}$ is a geometric Brownian motion and, moreover,

$$
V_{t}=\frac{S_{t}}{\rho-\mu} .
$$

Proof. We have

$$
V_{t}=\mathrm{E}_{t}\left[\int_{t}^{\infty} \mathrm{e}^{-\rho(u-t)} S_{t} \mathrm{e}^{\mu(u-t)} \exp \left\{-\frac{\sigma^{2}}{2}(u-t)+\sigma\left(Z_{u}-Z_{t}\right)\right\} \mathrm{d} u\right]
$$

where the last term is a martingale. Applying Fubini's theorem we obtain

$$
V_{t}=S_{t} \int_{t}^{\infty} \mathrm{e}^{-\rho(u-t)} \mathrm{e}^{\mu(u-t)} \mathrm{d} u=\frac{S_{t}}{\rho-\mu} .
$$

This completes the proof.

Therefore, $V_{t}$ satisfies the following stochastic differential equation:

$$
\frac{\mathrm{d} V_{t}}{V_{t}}=\mu \mathrm{d} t+\sigma Z_{t}, \quad V_{0}=\frac{S_{0}}{\rho-\mu} .
$$

Since the agents are risk neutral, the value of the investment and disinvestment decision problem can be written as a discounted expectation,

$$
\mathrm{E}\left[\exp \left\{-\rho \tau_{\mathrm{I}}\right\}\left(V_{\tau_{\mathrm{I}}}-C_{\mathrm{I}}\right)^{+}+\exp \left\{-\rho \tau_{\mathrm{D}}\right\}\left(C_{\mathrm{D}}-V_{\tau_{\mathrm{D}}}\right)^{+}\right]
$$

where $(\cdot)^{+}$corresponds to $\max (0, \cdot), C_{\mathrm{I}}$ and $C_{\mathrm{D}}$ respectively represent the investment and disinvestment costs, which we assume to be constant, and $\tau_{\mathrm{I}}$ and $\tau_{\mathrm{D}}$ represent the first instants when the process $V_{t}$ has consecutively spent $d$ units of time above or, respectively, below a specific threshold. This satisfies the Parisian criterion, i.e. the firm invests or disinvests at $\tau_{\mathrm{I}}$ or, respectively, $\tau_{\mathrm{D}}$ only if the decision variable $V_{t}$ has reached a prespecified level and has remained constantly above or, respectively, below this level for a time interval longer than a 


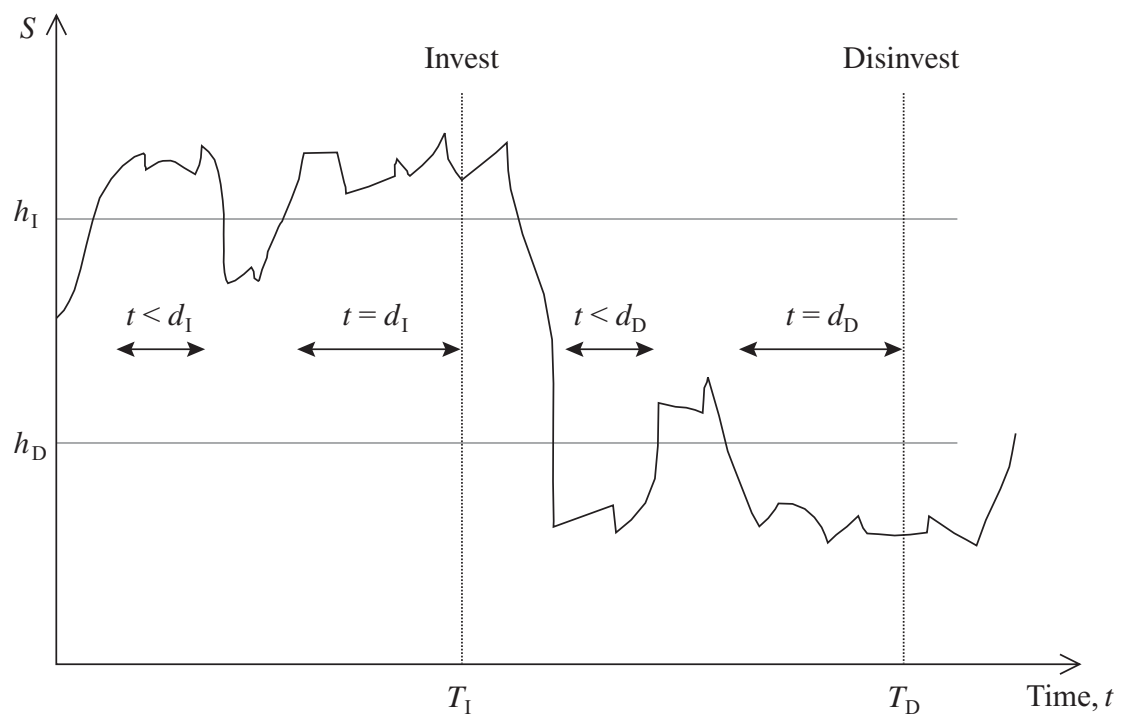

Figure 1: A possible sample path of the underlying process. After the process has consecutively spent $d_{\mathrm{I}}$ units of time above the investment threshold $h_{\mathrm{I}}^{*}$, the firm undertakes the investment. After the process has consecutively spent $d_{\mathrm{D}}$ units of time below the disinvestment threshold $h_{\mathrm{D}}^{*}$, the firm undertakes the disinvestment.

specific period of time (the so-called time window). See Figure 1 for a graphical interpretation. The time window corresponds to the implementation delay, whereas the prespecified levels correspond to the optimal investment threshold $h_{\mathrm{I}}^{*}$ and the optimal disinvestment threshold $h_{\mathrm{D}}^{*}$. We assume that the time window associated with the investment or disinvestment is a fixed amount of time $d_{\mathrm{I}}$ or, respectively, $d_{\mathrm{D}}$. The criterion that triggers the decision to act is the so-called Parisian stopping time which depends on the size of the excursions of the state variable above or below the optimal thresholds.

The firm maximizes the present value of its opportunities, namely, it solves the following problem:

$$
V F\left(V_{0}\right)=\max _{\tau_{\mathrm{I}}<\tau_{\mathrm{D}}} \mathrm{E}\left[\exp \left\{-\rho \tau_{\mathrm{I}}\right\}\left(V_{\tau_{\mathrm{I}}}-C_{\mathrm{I}}\right)^{+} \mathbf{1}_{\left\{\tau_{\mathrm{I}}<\infty\right\}}+\exp \left\{-\rho \tau_{\mathrm{D}}\right\}\left(C_{\mathrm{D}}-V_{\tau_{\mathrm{D}}}\right)^{+} \mathbf{1}_{\left\{\tau_{\mathrm{D}}<\infty\right\}}\right] .
$$

Because we are in the perpetual case, the investment or disinvestment decision will occur at the first instant when $V_{t}$ hits some constant optimal threshold $h_{\mathrm{I}}^{*}$ or, respectively, $h_{\mathrm{D}}^{*}$. Letting $\tau_{\mathrm{I}}$ and $\tau_{\mathrm{D}}$ be the stopping times which correspond to the Parisian criterion with time windows $d_{\mathrm{I}}$ and $d_{\mathrm{D}}$, and letting $h_{\mathrm{I}}$ and $h_{\mathrm{D}}$ respectively be the entry and exit levels, the present value of the investment and disinvestment decision problem becomes

$$
V F\left(V_{0}\right)=\max _{h_{\mathrm{D}} \leq h_{\mathrm{I}}, V_{0} \leq h_{\mathrm{I}}} \mathrm{E}_{0}\left[\exp \left\{-\rho \tau_{\mathrm{I}}\right\}\left(V_{\tau_{\mathrm{I}}}-C_{\mathrm{I}}\right) \mathbf{1}_{\left\{\tau_{\mathrm{I}}<\infty\right\}}+\exp \left\{-\rho \tau_{\mathrm{D}}\right\}\left(C_{\mathrm{D}}-V_{\tau_{\mathrm{D}}}\right) \mathbf{1}_{\left\{\tau_{\mathrm{D}}<\infty\right\}}\right] .
$$

\section{The solution of the problem}

In this section we solve the maximization problem, obtaining an analytic solution to the optimal entry and exit thresholds. Following the literature on Parisian options, we reformulate 
the problem in terms of the drifted Brownian motion. We define

$$
V_{t}=V_{0} \exp \left\{\sigma X_{t}\right\}, \quad \text { where } \quad X_{t}=b t+Z_{t} \text { and } b=\frac{\mu-\sigma^{2} / 2}{\sigma},
$$

and construct a new probability measure $\mathrm{P}^{*}$ under which $X_{t}$ becomes a $\mathrm{P}^{*}$-Brownian motion,

$$
\left.\frac{\mathrm{dP}^{*}}{\mathrm{dP}}\right|_{\mathcal{F}_{t}}=\exp \left\{\frac{b^{2}}{2} t-b X_{t}\right\} \text {. }
$$

Applying Girsanov's theorem, we change the probability measure in (2.2). Under the new measure, $\tau_{\mathrm{I}}<\infty$ and $\tau_{\mathrm{D}}<\infty$ hold almost surely. Using the independence result from Theorem C.5, we obtain

$$
\mathrm{E}_{\mathrm{P}^{*}}\left[\exp \left\{-\left(\rho+\frac{b^{2}}{2}\right) \tau_{\mathrm{I}}\right\}\right] \mathrm{E}_{\mathrm{P}^{*}}\left[\exp \left\{b X_{\tau_{\mathrm{I}}}\right\}\left(V_{0} \exp \left\{\sigma X_{\tau_{\mathrm{I}}}\right\}-C_{\mathrm{I}}\right)\right]
$$

for the first term in the maximization problem. Similarly, the second term becomes

$$
\mathrm{E}_{\mathrm{P}^{*}}\left[\exp \left\{-\left(\rho+\frac{b^{2}}{2}\right) \tau_{\mathrm{D}}\right\}\right] \mathrm{E}_{\mathrm{P}^{*}}\left[\exp \left\{b X_{\tau_{\mathrm{D}}}\right\}\left(C_{\mathrm{D}}-V_{0} \exp \left\{\sigma X_{\tau_{\mathrm{D}}}\right\}\right)\right] .
$$

In Appendix D we compute the Laplace transform of the Parisian investment and disinvestment times under the new measure $\mathrm{P}^{*}$ defined in (3.2). We calculate the moment generating function for the process $X_{t}$ defined in (3.1) and stopped at the Parisian investment and disinvestment times. Finally, we evaluate the first hitting time of $X$ which starts from the Parisian investment time. After that, and combining Proposition D.6 and Proposition D.7, we can rewrite the maximization problem, (2.2), as

$$
\begin{aligned}
V F\left(V_{0}\right)= & \max _{h_{\mathrm{D}} \leq h_{\mathrm{I}}, V_{0} \leq h_{\mathrm{I}}}\left(\frac{V_{0}}{h_{\mathrm{I}}}\right)^{\theta_{1}} \frac{\phi\left(b \sqrt{d_{\mathrm{I}}}\right)}{\phi\left(\sqrt{\left(2 \rho+b^{2}\right) d_{\mathrm{I}}}\right)}\left\{h_{\mathrm{I}} \frac{\phi\left(\sqrt{d_{\mathrm{I}}}(\sigma+b)\right)}{\phi\left(b \sqrt{d_{\mathrm{I}}}\right)}-C_{\mathrm{I}}\right. \\
& \left.+\left(\frac{h_{\mathrm{I}}}{h_{\mathrm{D}}}\right)^{\theta_{2}} \frac{\phi\left(-\sqrt{\left(2 \rho+b^{2}\right) d_{\mathrm{I}}}\right)}{\phi\left(\sqrt{\left(2 \rho+b^{2}\right) d_{\mathrm{D}}}\right)} \frac{\phi\left(-b \sqrt{d_{\mathrm{D}}}\right)}{\phi\left(b \sqrt{d_{\mathrm{I}}}\right)}\left(C_{\mathrm{D}}-h_{\mathrm{D}} \frac{\phi\left(-(b+\sigma) \sqrt{d_{\mathrm{D}}}\right)}{\phi\left(-b \sqrt{d_{\mathrm{D}}}\right)}\right)\right\},
\end{aligned}
$$

where $\phi(z)$ is the moment generating function derived in Appendix A and defined as

$$
\phi(z)=\int_{0}^{\infty} x \exp \left\{z x-\frac{1}{2} x^{2}\right\} \mathrm{d} x
$$

and where, to simplify the already complicated notation, we adopt the following:

$$
\theta_{1}=\frac{-b+\sqrt{2 \rho+b^{2}}}{\sigma} \text { and } \theta_{2}=\frac{-b-\sqrt{2 \rho+b^{2}}}{\sigma} .
$$

Let us start by considering the instantaneous investment and disinvestment problem, i.e. when $d_{\mathrm{I}}=d_{\mathrm{D}}=0$. First, we solve the unconstrained problem corresponding to (3.5). Taking its partial derivative with respect to $h_{\mathrm{D}}$ and solving for the critical value, we obtain an explicit solution for the optimal instantaneous disinvestment $h_{\mathrm{ND}}^{*}$,

$$
h_{\mathrm{ND}}^{*}=\frac{\theta_{2} C_{\mathrm{D}}}{\theta_{2}-1},
$$


whereas the optimal instantaneous investment threshold is $h_{\mathrm{NI}}^{*}=\max \left(V_{0}, x^{*}\right)$, where $x^{*}$ is the largest of the two solutions of the implicit equation

$$
x=\frac{\theta_{1} C_{\mathrm{I}}}{\theta_{1}-1}+\left(\frac{x}{h_{\mathrm{ND}}^{*}}\right)^{\theta_{2}} \frac{\theta_{2}-\theta_{1}}{\theta_{1}-1} \frac{C_{\mathrm{D}}}{1-\theta_{2}} ;
$$

the equation is obtained by taking the partial derivative of (3.5) with respect to $h_{\mathrm{I}}$ and solving for the critical value. However, the solution of the unconstrained maximization problem, $h_{\mathrm{ND}}^{*}$ and $\max \left(x^{*}\right)$, does not necessarily coincide with the solution of the constrained maximization problem. Imposing $C_{\mathrm{D}}<C_{\mathrm{I}}$, the solution satisfies the first constraint $h_{\mathrm{ND}}^{*} \leq \max \left(x^{*}\right)$. Assuming in addition that $V_{0} \leq h_{\mathrm{NI}}^{*}$, the (instantaneous) constrained problem has also correctly ordered optimal levels. Summarizing, the solution of the (instantaneous, i.e. $d_{\mathrm{I}}=d_{\mathrm{D}}=0$ ) unconstrained problem coincides with the solution of the constrained problem, imposing $C_{\mathrm{D}}<$ $C_{\mathrm{I}}$ and assuming that $V_{0} \leq h_{\mathrm{NI}}^{*}$.

We now consider the investment and disinvestment problem with Parisian delay for more general time windows, i.e. $d_{\mathrm{I}}>0$ and $d_{\mathrm{D}} \geq 0$. First, we solve the unconstrained problem corresponding to (3.5), where we assume that $V_{0} \leq h_{\mathrm{I}}$. This is a necessary assumption due to the mathematical construction of the Parisian investment stopping time that does not admit already started excursion (for further details see Appendix A and Appendix B). As we did for $h_{D} \leq h_{I}$, we could investigate under which conditions the constraint $V_{0} \leq h_{I}$ is implicitly satisfied. We leave this for future research. Taking its partial derivative with respect to $h_{\mathrm{D}}$ and solving for the critical value, we obtain an explicit solution for the optimal disinvestment threshold $h_{\mathrm{D}}^{*}$,

$$
h_{\mathrm{D}}^{*}=\frac{\phi\left(-b \sqrt{d_{\mathrm{D}}}\right)}{\phi\left(-(b+\sigma) \sqrt{d_{\mathrm{D}}}\right)} \frac{\theta_{2} C_{\mathrm{D}}}{\theta_{2}-1} .
$$

It is immediately observable that $h_{\mathrm{ND}}^{*}=h_{\mathrm{D}}^{*}$ when $d_{\mathrm{D}}=0$. Intuitively, $h_{\mathrm{D}}^{*}$ increases when the disinvestment cost $C_{\mathrm{D}}$ increases. Therefore, similar to the instantaneous investment and disinvestment problem, the higher the disinvestment cost the sooner the firm wants to exit. Furthermore, since $\phi$ is an increasing function, we obtain $h_{\mathrm{ND}}^{*} \leq h_{\mathrm{D}}^{*}$, meaning that the firm decides to disinvest earlier in the presence of a disinvestment delay.

Taking the partial derivative with respect to $h_{\mathrm{I}}$ and solving for the critical value, we obtain an implicit solution for $h_{\mathrm{I}}^{*}$, as in the case of the instantaneous investment and disinvestment problem. In particular, $h_{\mathrm{I}}^{*}=\max \left(V_{0}, x^{*}\right)$, where $x^{*}$ solves the implicit equation

$$
x=\frac{\theta_{1} C_{\mathrm{I}}}{\theta_{1}-1} \frac{\phi\left(b \sqrt{d_{\mathrm{I}}}\right)}{\phi\left((b+\sigma) \sqrt{d_{\mathrm{I}}}\right)}+\left(\frac{x}{h_{\mathrm{D}}^{*}}\right)^{\theta_{2}} \frac{\theta_{2}-\theta_{1}}{\theta_{1}-1} \frac{\phi\left(-\sqrt{\left(2 \rho+b^{2}\right) d_{\mathrm{I}}}\right)}{\phi\left(\sqrt{\left(2 \rho+b^{2}\right) d_{\mathrm{D}}}\right)} \frac{\phi\left(-b \sqrt{d_{\mathrm{D}}}\right)}{\phi\left((b+\sigma) \sqrt{d_{\mathrm{I}}}\right)} \frac{C_{\mathrm{D}}}{1-\theta_{2}} .
$$

Denoting the right-hand side of the implicit equation (3.7) by $f(x)$, we now prove that it has two solutions, one of which is larger than $h_{\mathrm{D}}^{*}$, if we impose condition (3.8), below.

Lemma 3.1. Let $f(x)$ be the right-hand side of (3.7), and let $h_{\mathrm{D}}^{*}$ be defined as in (3.6). Then the following relations hold.

(a) The function $f$ is increasing in $(0, \infty)$, and

$$
\lim _{x \searrow 0} f(x)=-\infty \quad \text { and } \quad \lim _{x \rightarrow \infty} f(x)=\frac{\theta_{1} C_{\mathrm{I}}}{\theta_{1}-1} \frac{\phi\left(b \sqrt{d_{\mathrm{I}}}\right)}{\phi\left((b+\sigma) \sqrt{d_{\mathrm{I}}}\right)} .
$$


(b) $f\left(h_{\mathrm{D}}^{*}\right)>h_{\mathrm{D}}^{*}$ if the following inequality holds:

$$
C_{\mathrm{D}} \frac{\phi\left(-b \sqrt{d_{\mathrm{D}}}\right)}{\phi\left(-(b+\sigma) \sqrt{d_{\mathrm{D}}}\right)}<C_{\mathrm{I}} \frac{\phi\left(b \sqrt{d_{\mathrm{I}}}\right)}{\phi\left((b+\sigma) \sqrt{d_{\mathrm{I}}}\right)} .
$$

Proof. Since $\theta_{1}>1$, part (a) follows easily. To prove part (b), note that the following relations hold since $\phi$ is an increasing function:

$$
\begin{aligned}
\frac{\theta_{1}}{\theta_{1}-1} & =\frac{1}{1-\theta_{2}}\left(-\theta_{2}+\frac{\theta_{1}-\theta_{2}}{\theta_{1}-1}\right) \\
& \geq \frac{1}{1-\theta_{2}}\left(-\theta_{2}+\frac{\theta_{1}-\theta_{2}}{\theta_{1}-1} \frac{\phi\left(-\sqrt{\left(2 \rho+b^{2}\right) d_{\mathrm{I}}}\right)}{\phi\left(\sqrt{\left(2 \rho+b^{2}\right) d_{\mathrm{D}}}\right)} \frac{\phi\left(-(b+\sigma) \sqrt{d_{\mathrm{D}}}\right)}{\phi\left((b+\sigma) \sqrt{d_{\mathrm{I}}}\right)}\right) .
\end{aligned}
$$

We now multiply the left- and right-hand side terms of the inequality above with the terms in (3.8) and we obtain

$$
\begin{aligned}
\frac{\theta_{1} C_{\mathrm{I}}}{\theta_{1}-1} \frac{\phi\left(b \sqrt{d_{\mathrm{I}}}\right)}{\phi\left((b+\sigma) \sqrt{d_{\mathrm{I}}}\right)}> & \frac{C_{\mathrm{D}}}{1-\theta_{2}} \frac{\phi\left(-b \sqrt{d_{\mathrm{D}}}\right)}{\phi\left(-(b+\sigma) \sqrt{d_{\mathrm{D}}}\right)} \\
& \times\left(-\theta_{2}+\frac{\theta_{1}-\theta_{2}}{\theta_{1}-1} \frac{\phi\left(-\sqrt{\left(2 \rho+b^{2}\right) d_{\mathrm{I}}}\right)}{\phi\left(\sqrt{\left(2 \rho+b^{2}\right) d_{\mathrm{D}}}\right)} \frac{\phi\left(-(b+\sigma) \sqrt{d_{\mathrm{D}}}\right)}{\phi\left((b+\sigma) \sqrt{d_{\mathrm{I}}}\right)}\right) .
\end{aligned}
$$

Regrouping the terms we obtain $f\left(h_{\mathrm{D}}^{*}\right)>h_{\mathrm{D}}^{*}$. This completes the proof.

Relying on the previous lemma, the implicit equation $x=f(x)$ has two solutions, termed $x_{1}^{*}$ and $x_{2}^{*}$, where $0<x_{1}^{*}<h_{\mathrm{D}}^{*}<x_{2}^{*}$. Hence, the unconstrained maximization version of (3.5) has two critical points: $\left(x_{1}^{*}, h_{\mathrm{D}}^{*}\right)$ and $\left(x_{2}^{*}, h_{\mathrm{D}}^{*}\right)$. Between them, only the second point satisfies the constraint $h_{\mathrm{D}}^{*}<x^{*}$. Therefore, assuming that $V_{0} \leq x_{2}^{*}$ and imposing condition (3.8), the critical point $\left(x_{2}^{*}, h_{\mathrm{D}}^{*}\right)$ is a local maximum point for the unconstrained maximization problem (3.5), and, thus, $\left(h_{\mathrm{I}}^{*}, h_{\mathrm{D}}^{*}\right)$ is the unique solution of (3.5). We summarize our results in the following theorem.

Theorem 3.1. Consider the investment and disinvestment decisions of a firm under the Parisian criterion with time windows $d_{\mathrm{I}}$ and $d_{\mathrm{D}}$. If (3.8) holds and $V_{0} \leq x_{2}^{*}$, then the optimal investment and disinvestment thresholds satisfy the following equations:

$$
h_{\mathrm{D}}^{*}=\frac{\phi\left(-b \sqrt{d_{\mathrm{D}}}\right)}{\phi\left(-(b+\sigma) \sqrt{d_{\mathrm{D}}}\right)} \frac{\theta_{2} C_{\mathrm{D}}}{\theta_{2}-1}
$$

and $h_{\mathrm{I}}^{*}=x^{*}$, where $x^{*}$ solves the implicit equation

$$
x=\frac{\theta_{1} C_{\mathrm{I}}}{\theta_{1}-1} \frac{\phi\left(b \sqrt{d_{\mathrm{I}}}\right)}{\phi\left((b+\sigma) \sqrt{d_{\mathrm{I}}}\right)}+\left(\frac{x}{h_{\mathrm{D}}^{*}}\right)^{\theta_{2}} \frac{\theta_{2}-\theta_{1}}{\theta_{1}-1} \frac{\phi\left(-\sqrt{\left(2 \rho+b^{2}\right) d_{\mathrm{I}}}\right)}{\phi\left(\sqrt{\left(2 \rho+b^{2}\right) d_{\mathrm{D}}}\right)} \frac{\phi\left(-b \sqrt{d_{\mathrm{D}}}\right)}{\phi\left((b+\sigma) \sqrt{d_{\mathrm{I}}}\right)} \frac{C_{\mathrm{D}}}{1-\theta_{2}} .
$$

\section{Model results}

We now present a brief discussion of the optimal investment and disinvestment thresholds, $h_{\mathrm{I}}^{*}$ and $h_{\mathrm{D}}^{*}$, in terms of the time windows $d_{\mathrm{I}}$ and $d_{\mathrm{D}}$.

(a) If $d_{\mathrm{I}}=d_{\mathrm{D}}=0$, we recover the well-known case of the instantaneous investment and disinvestment problem and, therefore, $h_{\mathrm{I}}^{*}=h_{\mathrm{NI}}^{*}$ and $h_{\mathrm{D}}^{*}=h_{\mathrm{ND}}^{*}$. 
TABLE 1: Ratio of the Parisian value problem to the instantaneous value problem. The parameters are

\begin{tabular}{cccccc}
$\rho=0.13, \mu=0.05, \sigma=0.40$, & $C_{\mathrm{D}}=0.5, C_{\mathrm{I}}=1.7$, and $V_{0}=1$ \\
\hline \multirow{2}{*}{$d_{\mathrm{I}}$} & \multicolumn{5}{c}{$d_{\mathrm{D}}$} \\
\cline { 2 - 6 } & 0.0 & 0.5 & 1.0 & 1.5 & 2.0 \\
\hline 0.0 & 1.0000 & 0.9999 & 0.9998 & 0.9997 & 0.9996 \\
0.5 & 0.9872 & 0.9871 & 0.9870 & 0.9869 & 0.9868 \\
1.0 & 0.9738 & 0.9737 & 0.9736 & 0.9735 & 0.9734 \\
1.5 & 0.9600 & 0.9599 & 0.9598 & 0.9598 & 0.9597 \\
2.0 & 0.9461 & 0.9460 & 0.9459 & 0.9458 & 0.9457 \\
\hline
\end{tabular}

(b) If $d_{\mathrm{D}}=0$ and $d_{\mathrm{I}} \geq 0$, then $h_{\mathrm{D}}^{*}=h_{\mathrm{ND}}^{*}$.

(c) If $d_{\mathrm{D}} \rightarrow \infty$ then $h_{\mathrm{I}}^{*}$ converges to

$$
h_{\mathrm{OI}}^{*}=\frac{\theta_{1} C_{\mathrm{I}}}{\theta_{1}-1} \frac{\phi\left(b \sqrt{d_{\mathrm{I}}}\right)}{\phi\left((b+\sigma) \sqrt{d_{\mathrm{I}}}\right)},
$$

where $h_{\mathrm{OI}}^{*}$ represents the optimal investment threshold for the time window $d_{\mathrm{I}}$, while disinvestment is not possible. Gauthier and Morellec (2000) were the first to obtain this result.

(d) If $d_{\mathrm{D}} \geq 0$ and $d_{\mathrm{I}} \geq 0$, then $h_{\mathrm{D}}^{*} \geq h_{\mathrm{ND}}^{*}$.

For illustrative purposes, we perform a numerical evaluation to compare the investment and disinvestment decision problem in the presence of implementation delay and the instantaneous investment and disinvestment decision problem. In Table 1 we report the ratio of the value of the Parisian investment and disinvestment decision problem with respect to the instantaneous investment and disinvestment decision problem, at their respective optima. Since the first column and row correspond to the values of $d_{\mathrm{I}}$ and $d_{\mathrm{D}}$, respectively, we expect this ratio to be equal to 1 when the delay $d_{\mathrm{I}}=d_{\mathrm{D}}=0$. This is the case, as we observe in the upper-left corner of Table 1.

We observe that the value of the investment and disinvestment decision problem is lower under the Parisian criterion than in the instantaneous case for reasonable parameter values. This is expected, because the time lag under the Parisian criterion measures a systematic (and unavoidable) delay that forces the firm to 'postpone' the investment (or disinvestment) procedure. Of particular interest is the presence of an asymmetric effect: the larger $d_{\mathrm{I}}$, the stronger the impact on the investment value. Conversely, $d_{\mathrm{D}}$ does not have such a strong impact, possibly due to the reversibility of the investment decision. Since firm's profits are a convex function of the stochastic underlying and disinvestment is possible at a cost, a firm will invest at a lower level when the implementation delay forces it to decide in advance whether to enter a few periods ahead or not. In other words, the impact of $d_{\mathrm{I}}$ dominates $d_{\mathrm{D}}$.

Since either instantaneous and Parisian investment/disinvestment optima are linear functions of both $C_{\mathrm{D}}$ and $C_{\mathrm{I}}$, only the impact on the optimal thresholds of the volatility of the underlying process requires further investigation. Our findings confirm the results of numerous papers which report that an increase in uncertainty delays (instantaneous) investments. (See Pindyck (1991) for a survey.) The first row of Table 2 shows the effect of an increase in uncertainty without investment delay. As $\sigma$ goes from 0.05 to $0.40, h_{\mathrm{I}}^{*}$ rises from 2.8291 to 5.3185 , while $h_{\mathrm{D}}^{*}$ 
TABLE 2: Parisian optimal investment value $h_{\mathrm{I}}^{*}$. The parameters are $\rho=0.13, \mu=0.05, C_{\mathrm{D}}=0.5$, $C_{\mathrm{I}}=1.7$, and $V_{0}=1$.

\begin{tabular}{lccccccccc}
\hline & \multicolumn{3}{c}{$\sigma=0.05$} & \multicolumn{3}{c}{$\sigma=0.20$} & \multicolumn{3}{c}{$\sigma=0.40$} \\
\cline { 2 - 10 }$d_{\mathrm{I}}$ & \multicolumn{3}{c}{0.0 .0} & 0.5 & 1.0 & 0.0 & 0.5 & 1.0 \\
\cline { 2 - 10 } & 0.0 & 0.5 & 1.0 & 0.0 & & \multicolumn{3}{c}{$d_{\mathrm{D}}$} \\
\hline 0 & 2.8291 & 2.8291 & 2.8291 & 3.5735 & 3.5735 & 3.5734 & 5.3185 & 5.3113 & 5.3080 \\
3 & 2.3221 & 2.3221 & 2.3221 & 2.1571 & 2.1571 & 2.1570 & 2.0858 & 2.0823 & 2.0807 \\
5 & 2.0973 & 2.0973 & 2.0973 & 1.8080 & 1.8079 & 1.8079 & 1.5447 & 1.5417 & 1.5403 \\
\hline
\end{tabular}

falls from 0.4882 to 0.2620 (not reported in the table). The higher (instantaneous) investment threshold and the lower (instantaneous) disinvestment threshold imply that further uncertainty delays both entry and exit, and, thus, generates more so-called inertia. The intuition behind such a conventional result is that a firm delays in order to avoid learning bad news after it has made its decision to enter or exit. The likelihood of receiving bad news rises with uncertainty, as does the benefit of waiting. However, waiting has an opportunity cost due to the loss of income during the period of inaction and this is more evident in the presence of delays. As a result, conventional findings on the effect of the uncertainty of the underlying process on the investment and disinvestment are reversed when there are time lags. Similar to Bar-Ilan and Strange (1996), Table 2 shows that an increase in uncertainty hastens the decision to invest and disinvest. For instance, when $d_{\mathrm{I}}=5, h_{\mathrm{I}}^{*}$ falls from 2.0973 to 1.5447 , while $h_{\mathrm{D}}^{*}$ rises from 0.5201 to 0.5884 (not reported in the table). Since a firm can exit at a cost, the downside risk of the project is bounded. This makes profits a convex function of the stochastic underlying, and the expected return of the project rises with uncertainty. Therefore, a higher volatility hastens investment and disinvestment when delays force a firm to decide in advance whether or not to undertake a decision in the near future.

An interesting direction for future research would be the analytical study of the behavior of optimal thresholds in the Parisian decision problem as functions of the delays $d_{\mathrm{I}}$ and $d_{\mathrm{D}}$. Moreover, we can look for explicit conditions when inequality (3.8) holds. Although possible, such an analysis would require a careful study of the properties of the function $\phi$.

\section{Appendix A. Definitions and results}

The Brownian meander and the Parisian criterion are closely related. In the following we define the Brownian meander and list some of its properties. Then, we present the connection existing between the Brownian meander and the Parisian criterion.

Let $\left(Z_{t}, t \geq 0\right)$ be a standard Brownian motion on a filtered probability space $(\Omega, \mathcal{F}$, $\left.\left(\mathcal{F}_{t}\right)_{t \geq 0}, \mathrm{P}\right)$. For each $t>0$, we define the random variables

$$
\begin{aligned}
& g_{t}=\sup \left\{s: s \leq t, Z_{s}=0\right\} \\
& d_{t}=\inf \left\{s: s \geq t, Z_{s}=0\right\}
\end{aligned}
$$

The interval $\left(g_{t}, d_{t}\right)$ is called the 'interval of the Brownian excursion' which straddles time $t$. For $u$ in this interval, $\operatorname{sgn}\left(Z_{t}\right)$ remains constant. In particular, $g_{t}$ represents the last time the Brownian motion crossed the level 0. It is known that $g_{t}$ is not a stopping time for the Brownian filtration $\left(\mathcal{F}_{t}\right)_{t \geq 0}$ but for the slow Brownian filtration $\left(g_{t}\right)_{t \geq 0}$, which is defined 
by $g_{t}=\mathcal{F}_{g_{t}} \vee \sigma\left(\operatorname{sgn}\left(Z_{t}\right)\right)$. The slow Brownian filtration represents the information on the Brownian motion until its last 0 plus the knowledge of its sign after this.

The Brownian meander process ending at $t$ is defined as

$$
m_{u}^{(t)}=\frac{1}{\sqrt{t-g_{t}}}\left|Z_{g_{t}+u\left(t-g_{t}\right)}\right|, \quad 0 \leq u \leq 1 .
$$

The process $m_{u}^{(t)}$ is the nonnegative and normalized Brownian excursion which straddles time $t$ and is independent of the $\sigma$-field $\left(g_{t}\right)_{t \geq 0}$. When $u=1$ and $t=1$, we conveniently denote $m_{1}=m_{1}^{(1)}$. The random variable $m_{1}$ will play a central role in the calculation of many other variables that will be introduced later on. The distribution of $m_{1}$ is known to be

$$
\mathrm{P}\left(m_{1} \in \mathrm{d} x\right)=x \exp \left\{-\frac{1}{2} x^{2}\right\} \mathbf{1}_{x>0} \mathrm{~d} x,
$$

and the moment generating function $\phi(z)$ is given by

$$
\phi(z)=\mathrm{E}\left[\exp \left\{z m_{1}\right\}\right]=\int_{0}^{\infty} x \exp \left\{z x-\frac{1}{2} x^{2}\right\} \mathrm{d} x .
$$

We now look at the first instant when the Brownian motion spends $d$ units of time consecutively above or below the level 0 . For $d \geq 0$, we define the random variables

$$
\begin{aligned}
& H_{d}^{+}=\inf \left\{t \geq 0: t-g_{t} \geq d, Z_{t} \geq 0\right\}, \\
& H_{d}^{-}=\inf \left\{t \geq 0: t-g_{t} \geq d, Z_{t} \leq 0\right\} .
\end{aligned}
$$

The variables $H_{d}^{+}$and $H_{d}^{-}$are $g_{t}$-stopping times and, hence, $\mathcal{F}_{t}$-stopping times (see Revuz and Yor (1991, Chapter XII) for more details). From (A.1) we can easily deduce that the process

$$
\left(\frac{1}{\sqrt{d}}\left|Z_{g_{H_{d}^{+}}+u d}\right|\right)_{u \leq 1}=\left(m_{u}^{\left(H_{d}^{+}\right)}\right)_{u \leq 1}
$$

is a Brownian meander, independent of $g_{g_{H_{d}^{+}}}$. In particular, $(1 / \sqrt{d}) Z_{H_{d}^{+}}$is distributed as $m_{1}$,

$$
\mathrm{P}\left(Z_{H_{d}^{+}} \in \mathrm{d} x\right)=\frac{x}{d} \exp \left\{-\frac{x^{2}}{2 d}\right\} \mathbf{1}_{x>0} \mathrm{~d} x,
$$

and the random variables $H_{d}^{+}$and $Z_{H_{d}^{+}}$are independent.

Similarly, $(1 / \sqrt{d}) Z_{H_{d}^{-}}$is distributed as $-m_{1}$,

$$
\mathrm{P}\left(Z_{H_{d}^{-}} \in \mathrm{d} x\right)=\frac{-x}{d} \exp \left\{-\frac{x^{2}}{2 d}\right\} \mathbf{1}_{x<0} \mathrm{~d} x,
$$

and the random variables $H_{d}^{-}$and $Z_{H_{d}^{-}}$are independent.

Chesney et al. (1997) were the first to calculate the Laplace transform of $H_{d}^{+}$. We present the result in the next theorem.

Theorem A.1. Let $H_{d}^{+}$be the stopping time defined in (A.3), and let $\phi$ be the moment generating function defined in (A.2). For any $\lambda>0$,

$$
\mathrm{E}\left[\exp \left\{-\lambda H_{d}^{+}\right\}\right]=\frac{1}{\phi(\sqrt{2 \lambda d})} .
$$


The proof is based on the Azèma martingale, $\mu_{t}=\operatorname{sgn}\left(Z_{t}\right) \sqrt{t-g_{t}}$, a remarkable $\left(g_{t}\right)$ martingale. The same results hold also when $H_{d}^{+}$is replaced with $H_{d}^{-}$.

So far we have only looked at the Brownian motion excursions above or below level 0 . More generally, we can define, for any $a \in \mathbb{R}$ and any continuous stochastic process $X$,

$$
\begin{aligned}
g_{t}^{X_{0}, a}(X) & =\sup \left\{s: s \leq t, X_{t}=a \mid X_{0}=x\right\}, \\
H_{\left(X_{0}, a\right), d}^{+}(X) & =\inf \left\{t \geq 0: t-g_{t}^{X_{0}, a} \geq d, X_{t} \geq a \mid X_{0}=x\right\}, \\
H_{\left(X_{0}, a\right), d}^{-}(X) & =\inf \left\{t \geq 0: t-g_{t}^{X_{0}, a} \geq d, X_{t} \leq a \mid X_{0}=x\right\} .
\end{aligned}
$$

Thus, $g_{t}^{X_{0}, a}(X)$ represents the last time the process $X$ crossed level $a$. As for the Brownian motion case, $g_{t}^{X_{0}, a}(X)$ is not a stopping time for the Brownian filtration $\left(\mathcal{F}_{t}\right)_{t \geq 0}$ but for the slow Brownian filtration $\left(g_{t}\right)_{t \geq 0}$. The random variables $H_{\left(X_{0}, a\right), d}^{+}(X)$ and $H_{\left(X_{0}, a\right), d}^{-}(X)$ represent the first instants when the process $X$ spends $d$ units of time above or, respectively, below the level $a$. The variables $H_{\left(X_{0}, a\right), d}^{+}(X)$ and $H_{\left(X_{0}, a\right), d}^{-}(X)$ are $g_{t}$-stopping times and, hence, $\mathcal{F}_{t}$-stopping times. In the notation we use we indicate the starting point of the process $X$, the level $a$, and the length of time $d$. Although indicating the starting point seems unnecessary, it turns out to be extremely helpful in the context of the Parisian criterion.

Another relevant random variable is the first hitting time of level $a$, which we define as

$$
T_{X_{0}, a}(X)=\inf \left\{s: X_{s}=a \mid X_{0}=x\right\} .
$$

\section{Appendix B. Parisian criterion}

According to the notation introduced in Section 2, the investment stopping time $\tau_{\text {I }}$ which satisfies the Parisian criterion corresponds to $H_{\left(V_{0}, h_{\mathrm{I}}\right), d_{\mathrm{I}}}^{+}(V)$. In order to express the disinvestment stopping time $\tau_{\mathrm{D}}$ in mathematical formulae, we need to extend the definition of $H_{\left(V_{0}, h_{\mathrm{I}}\right), d_{\mathrm{I}}}^{+}(V)$. Let $\tau$ be any stopping time, let $a \in \mathbb{R}$, let $X$ be a continuous stochastic process, and let $g_{t}^{X_{0}, a}(X)$ be as defined in (A.4). Then

(a) the first instant after $\tau$ when the process $X$ spends $d$ units of time above or below level $a$ is given by the stopping time $H_{\left(X_{0}, a\right), d}^{+, \tau}(X)$ or, respectively, $H_{\left(X_{0}, a\right), d}^{-, \tau}(X)$ :

$$
\begin{aligned}
& H_{\left(X_{0}, a\right), d}^{+, \tau}(X)=\inf \left\{t \geq \tau: t-g_{t}^{X_{0}, a} \geq d, X_{t} \geq a \mid X_{0}=x\right\}, \\
& H_{\left(X_{0}, a\right), d}^{-, \tau}(X)=\inf \left\{t \geq \tau: t-g_{t}^{X_{0}, a} \geq d, X_{t} \leq a \mid X_{0}=x\right\} ;
\end{aligned}
$$

(b) the first hitting time after $\tau$ of level $a$ is the stopping time $T_{X_{0}, a}^{\tau}(X)$,

$$
T_{X_{0}, a}^{\tau}(X)=\inf \left\{s \geq \tau: X_{s}=a \mid X_{0}=x\right\} .
$$

If $X$ has the strong Markov property and $\tau$ is a finite stopping time, we have the following equalities in distribution: $H_{\left(X_{0}, a\right), d}^{+, \tau}(X)=H_{\left(X_{\tau}, a\right), d}^{+}(X), H_{\left(X_{0}, a\right), d}^{-, \tau}(X)=H_{\left(X_{\tau}, a\right), d}^{-}(X)$, and $T_{X_{0}, a}^{\tau}(X)=T_{X_{\tau}, a}(X)$. Now we can state the formulae for the stopping times $\tau_{\mathrm{I}}$ and $\tau_{\mathrm{D}}$, which satisfy the Parisian criterion.

Proposition B.1. Let $\tau_{\mathrm{I}}$ and $\tau_{\mathrm{D}}$ be the stopping times corresponding to the Parisian criterion with time windows $d_{\mathrm{I}}$ and $d_{\mathrm{D}}$ and levels $h_{\mathrm{I}}$ and $h_{\mathrm{D}}$, respectively. Then the following equalities hold:

$$
\tau_{\mathrm{I}}=H_{\left(V_{0}, h_{\mathrm{I}}\right), d_{\mathrm{I}}}^{+}(V), \quad \tau_{\mathrm{D}}=H_{\left(V_{0}, h_{\mathrm{D}}\right), d_{\mathrm{D}}}^{-, \tau_{\mathrm{I}}}(V)
$$


Otherwise, in terms of the drifted Brownian motion, the Parisian stopping times are

$$
\tau_{\mathrm{I}}=H_{\left(V_{0}, h_{\mathrm{I}}\right), d_{\mathrm{I}}}^{+}(V)=H_{\left(l_{0}, l_{\mathrm{I}}\right), d_{\mathrm{I}}}^{+}(X), \quad \text { where } \quad l_{0}=0 \text { and } l_{\mathrm{I}}=\frac{1}{\sigma} \log \left(\frac{h_{\mathrm{I}}}{V_{0}}\right),
$$

and

$$
\tau_{\mathrm{D}}=H_{\left(V_{0}, h_{\mathrm{D}}\right), d_{\mathrm{D}}}^{-, \tau_{\mathrm{I}}}(V)=H_{\left(l_{0}, l_{\mathrm{D}}\right), d_{\mathrm{D}}}^{-, \tau_{\mathrm{I}}}(X), \quad \text { where } \quad l_{0}=0 \text { and } l_{\mathrm{D}}=\frac{1}{\sigma} \log \left(\frac{h_{\mathrm{D}}}{V_{0}}\right) .
$$

\section{Appendix C. Parisian stopping times and independence}

In this appendix we prove that the Parisian investment and disinvestment times and the position of the underlying value process at that time are independent. We rely on the key property of independence of the Brownian meander from the slow Brownian filtration. The independence is a pivotal result that allows us to perform exact calculations of the maximization problem, (2.2), in Section 3.

The following proposition helps us to decompose the disinvestment Parisian time, provided that we have proved the independence relationship between the Parisian investment time and the position of the underlying value process.

Proposition C.1. Let $\tau$ be any finite stopping time such that $\tau$ and $V_{\tau}$ are independent, and assume that $h_{\mathrm{D}} \leq V_{\tau}$ almost surely (a.s.). Then the following equality in distribution holds:

$$
H_{\left(V_{0}, h_{\mathrm{D}}\right), d_{\mathrm{D}}}^{-, \tau}(V)=\tau+T_{V_{\tau}, h_{\mathrm{D}}}(V)+H_{\left(h_{\mathrm{D}}, h_{\mathrm{D}}\right), d_{\mathrm{D}}}^{-}(V),
$$

and the terms of the sum are independent. A similar relationship holds for $H_{\left(V_{0}, h_{\mathrm{I}}\right), d_{\mathrm{I}}}^{+, \tau}(V)$ if we assume that $V_{\tau} \leq h_{\mathrm{I}}$ a.s.

Proof. The strong Markov property and the continuity of the process $V$ give us the equality. The independence follows from our hypothesis that $\tau$ and $V_{\tau}$ are independent.

The next theorem shows that the Parisian disinvestment time and the position of the underlying value process at that time are independent.

Theorem C.1. Assume that $h_{\mathrm{D}} \leq h_{\mathrm{I}}$ and that $V_{0} \leq h_{\mathrm{I}}$, let $\tau_{\mathrm{D}}=H_{\left(V_{0}, h_{\mathrm{D}}\right), d_{\mathrm{D}}}^{-, \tau_{\mathrm{I}}}(V)$, and let $\mathrm{P}^{*}$ be as defined in (3.2). Then the stopping time $\tau_{\mathrm{D}}$ is finite $\mathrm{P}^{*}$-a.s. and the random variables $\tau_{\mathrm{D}}$ and $V_{\tau_{\mathrm{D}}}$ are independent under the $\mathrm{P}^{*}$-measure.

The proof is broken down into several steps and relies on the next lemma. The lemma is one of the main results of this paper and it proves an interesting independence property of the Brownian motion with drift.

Lemma C.1. Let $X_{t}=b t+Z_{t}$, where $b \in \mathbb{R}$, and construct the stopping time $T=H_{(0,0), d}^{+}(X)$ according to (A.4). The following conclusions hold.

(a) The random variables $X_{T} \mathbf{1}_{\{T<\infty\}}$ and $T \mathbf{1}_{\{T<\infty\}}$ are independent under the $\mathrm{P}$-measure if and only if $b \geq 0$.

(b) The random variables $X_{T} \mathbf{1}_{\{T<\infty\}}$ and $T \mathbf{1}_{\{T<\infty\}}$ are independent under the $\mathrm{P}^{*}$-measure for any $b \in \mathbb{R}$.

A similar relationship holds when $H_{(0,0), d}^{+}(X)$ is replaced with $H_{(0,0), d}^{-}(X)$. 
Proof. Using Girsanov's theorem, we construct the probability measure $\mathrm{P}^{*}$ under which $X_{t}$ becomes a $\mathrm{P}^{*}$-Brownian motion. Under this probability, $X_{T}=X_{H_{(0,0), d}^{+}(X)}$ is a Brownian meander and, thus, it is independent of $T=H_{(0,0), d}^{+}(X)$. Also, $\mathbf{1}_{\{T<\infty\}} \stackrel{(0,0), d}{=} 1$ a.s. under the measure $\mathrm{P}^{*}$. Thus, we have the required independence under the $\mathrm{P}^{*}$-measure for any $b \in \mathbb{R}$. We need to show that the independence also holds under the original measure $\mathrm{P}$ if and only if $b \geq 0$.

The independence holds if and only if the Laplace transforms satisfy the equality

$$
\mathrm{E}_{\mathrm{P}}\left[\exp \left\{-\lambda X_{T}-\alpha T\right\} \mathbf{1}_{\{T<\infty\}}\right]=\mathrm{E}_{\mathrm{P}}\left[\exp \left\{-\lambda X_{T}\right\} \mathbf{1}_{\{T<\infty\}}\right] \mathrm{E}_{\mathrm{P}}\left[\mathrm{e}^{-\alpha T} \mathbf{1}_{\{T<\infty\}}\right] .
$$

Hence, we show that the equality holds if and only if $b \geq 0$.

Let us apply a transformation of measure to the left-hand side of (C.1). (We thank an anonymous referee for the derivation of the Girsanov's transformation with $T$ being a stopping time.) Let us consider a fixed time $T^{*}$. Then

$$
\begin{aligned}
\mathrm{E}_{\mathrm{P}}\left[\exp \left\{-\lambda X_{T}-\alpha T\right\} \mathbf{1}_{\left\{T \leq T^{*}\right\}}\right]= & \mathrm{E}_{\mathrm{P}^{*}}\left[\exp \left\{-\lambda X_{T}-\alpha T\right\} \mathbf{1}_{\left\{T \leq T^{*}\right\}} \exp \left\{-\frac{1}{2} b^{2} T^{*}+b X_{T^{*}}\right\}\right] \\
= & \mathrm{E}_{\mathrm{P}^{*}}\left[\exp \left\{-\lambda X_{T}-\alpha T\right\} \mathbf{1}_{\left\{T \leq T^{*}\right\}}\right. \\
& \left.\quad \times \mathrm{E}_{\mathrm{P}^{*}}\left[\exp \left\{-\frac{1}{2} b^{2} T^{*}+b X_{T^{*}}\right\} \mid X_{T}, T\right]\right] \\
= & \mathrm{E}_{\mathrm{P}^{*}}\left[\exp \left\{-\lambda X_{T}-\alpha T\right\} \mathbf{1}_{\left\{T \leq T^{*}\right\}} \exp \left\{-\frac{1}{2} b^{2} T+b X_{T}\right\}\right] .
\end{aligned}
$$

This relies on the Markov property and the fact that $\exp \left\{-b^{2} T / 2+b X_{T}\right\}$ is a $\mathrm{P}^{*}$-martingale. Now, setting $T^{*} \rightarrow \infty$ and because, under the $\mathrm{P}^{*}$-measure, $\mathbf{1}_{\{T<\infty\}}=1$ a.s., we obtain

$$
\mathrm{E}_{\mathrm{P}}\left[\exp \left\{-\lambda X_{T}-\alpha T\right\} \mathbf{1}_{\{T<\infty\}}\right]=\mathrm{E}_{\mathrm{P} *}\left[\exp \left\{-\lambda X_{T}-\alpha T\right\} \exp \left\{-\frac{1}{2} b^{2} T+b X_{T}\right\}\right] .
$$

We know that, under the $\mathrm{P}^{*}$-measure, $X_{T}$ is independent of $T$ and, grouping the factors, the left-hand side of (C.1) becomes

$$
\mathrm{E}_{\mathrm{P}}\left[\exp \left\{-\lambda X_{T}-\alpha T\right\} \mathbf{1}_{\{T<\infty\}}\right]=\mathrm{E}_{\mathrm{P} *}\left[\exp \left\{(-\lambda+b) X_{T}\right\}\right] \mathrm{E}_{\mathrm{P}}\left[\exp \left\{\left(-\alpha-\frac{1}{2} b^{2}\right) T\right\}\right] .
$$

Let us now calculate the product of the right-hand side of (C.1). Using Girsanov's theorem, we change the measure in both terms:

$$
\mathrm{E}_{\mathrm{P}^{*}}\left[\exp \left\{-\lambda X_{T}\right\} \exp \left\{-\frac{1}{2} b^{2} T+b X_{T}\right\}\right] \mathrm{E}_{\mathrm{P}^{*}}\left[\mathrm{e}^{-\alpha T} \exp \left\{-\frac{1}{2} b^{2} T+b X_{T}\right\}\right] .
$$

Since, under the $\mathrm{P}^{*}$-measure, $X_{T}$ is independent of $T$, we obtain

$$
\mathrm{E}_{\mathrm{P}^{*}}\left[\exp \left\{(-\lambda+b) X_{T}\right\}\right] \mathrm{E}_{\mathrm{P}^{*}}\left[\exp \left\{\left(-\alpha-\frac{1}{2} b^{2}\right) T\right\}\right] \mathrm{E}_{\mathrm{P}^{*}}\left[\exp \left\{-\frac{1}{2} b^{2} T+b X_{T}\right\}\right] .
$$

Therefore, we can immediately identify the extra term

$$
\mathrm{E}_{\mathrm{P}^{*}}\left[\exp \left\{-\frac{1}{2} b^{2} T+b X_{T}\right\}\right]=\frac{\phi(b \sqrt{d})}{\phi(|b| \sqrt{d})},
$$

where the last equality follows from the properties of the Brownian meander. This extra term is equal to 1 if and only if $b \geq 0$, which means that the independence holds if and only if $b \geq 0$.

Remark. It is worth noting that in Chesney et al. (1997) all computations are carried out under the measure $\mathrm{P}^{*}$, where the independence comes automatically from the properties of the Brownian meander. In Gauthier and Morellec (2000) the authors do not investigate under which conditions for $b$ the independence under the measure $\mathrm{P}$ holds. 
Analyzing the independence relationships we can observe an interesting connection with Wald's identity. Let us recall the following known theorem which relates Wald's identity to the finiteness of the stopping times.

Theorem C.2. Let $Z_{t}$ be a P-Brownian motion, and let $X_{t}=b t+Z_{t}$ be a drifted $\mathrm{P}$-Brownian motion. Let $\mathrm{P}^{*}$ be the measure under which $X_{t}$ is a $\mathrm{P}^{*}$-Brownian motion. Let $T$ be any stopping time, and assume that $\mathrm{P}^{*}(T<\infty)=1$. Then Wald's identity,

$$
\mathrm{E}_{\mathrm{P} *}\left[\exp \left\{-\frac{1}{2} b^{2} T+b X_{T}\right\}\right]=1,
$$

holds if and only if $\mathrm{P}(T<\infty)=1$.

In our case, $T=H_{(0,0), d}^{+}(X)$. From Chesney et al. (1997) we know that $\mathrm{P}^{*}(T<\infty)=1$ and, based on the properties of the Brownian meander, we also know that

$$
\mathrm{E}_{\mathrm{P} *}\left[\exp \left\{-\frac{1}{2} b^{2} T+b X_{T}\right\}\right]=1 \quad \text { if and only if } \quad b \geq 0 .
$$

Thus, $\mathrm{P}(T<\infty)<1$ if and only if $b<0$. Let us now compute $\mathrm{P}(T<\infty)$. In order to proceed, we first take a detour and calculate the Laplace transform of $H_{(0,0), d}^{+}(X)$ under the P-measure.

Theorem C.3. Let $X_{t}=b t+Z_{t}$, where $b$ is some fixed real number, and construct the stopping time $H_{(0,0), d}^{+}(X)$ according to (A.4). Let $\phi$ be the moment generating function defined in (A.2). Then, for any $\lambda>0$, the Laplace transform of $H_{(0,0), d}^{+}(X)$ is given by

$$
\mathrm{E}_{\mathrm{P}}\left[\exp \left\{-\lambda H_{(0,0), d}^{+}(X)\right\}\right]=\frac{\phi(b \sqrt{d})}{\phi\left(\sqrt{\left(2 \lambda+b^{2}\right) d}\right)} .
$$

Proof. Using Girsanov's theorem, we construct the probability measure $\mathrm{P}^{*}$ under which $X_{t}$ becomes a $\mathrm{P}^{*}$-Brownian motion. Under this probability, $X_{H_{(0,0), d}^{+}(X)}$ becomes a Brownian meander and, thus, it is independent of $H_{(0,0), d}^{+}(X)$. We then compute the Laplace transform and apply the change of measure

$$
\begin{aligned}
\mathrm{E}_{\mathrm{P}}\left[\exp \left\{-\lambda H_{(0,0), d}^{+}(X)\right\}\right]=\mathrm{E}_{\mathrm{P}^{*}}[ & \exp \left\{-\lambda H_{(0,0), d}^{+}(X)\right\} \\
& \left.\times \exp \left\{-\frac{1}{2} b^{2} H_{(0,0), d}^{+}(X)+b X_{H_{(0,0), d}^{+}(X)}\right\}\right] .
\end{aligned}
$$

Grouping terms together and using the independence property, we obtain

$$
\mathrm{E}_{\mathrm{P}}\left[\exp \left\{-\lambda H_{(0,0), d}^{+}(X)\right\}\right]=\mathrm{E}_{\mathrm{P}^{*}}\left[\exp \left\{\left(-\lambda-\frac{1}{2} b^{2}\right) H_{(0,0), d}^{+}(X)\right\}\right] \mathrm{E}_{\mathrm{P}^{*}}\left[\exp \left\{b X_{H_{(0,0), d}^{+}(X)}\right\}\right] .
$$

Using the Laplace transform of the Brownian meander and recognizing that $\phi$ is the moment generating function of $(1 / \sqrt{d}) H_{(0,0), d}^{+}(X)$, we obtain

$$
\mathrm{E}_{\mathrm{P}}\left[\exp \left\{-\lambda H_{(0,0), d}^{+}(X)\right\}\right]=\frac{\phi(b \sqrt{d})}{\phi\left(\sqrt{\left(2 \lambda+b^{2}\right) d}\right)} .
$$

This completes the proof.

We now calculate $\mathrm{P}(T<\infty)$, where we let $T=H_{(0,0), d}^{+}(X)$. If $T(\omega)<\infty$ then

$$
\lim _{\lambda \searrow 0} \mathrm{e}^{-\lambda T(\omega)}=1
$$


if $T(\omega)=\infty$ then $\mathrm{e}^{-\lambda T(\omega)}=0$ for every $\lambda>0$, so

$$
\lim _{\lambda \searrow 0} \mathrm{e}^{-\lambda T(\omega)}=0
$$

Therefore,

$$
\lim _{\lambda \searrow 0} \mathrm{e}^{-\lambda T(\omega)}=\mathbf{1}_{\{T<\infty\}}
$$

Letting $\lambda \searrow 0$ and using the monotone convergence theorem, we obtain

$$
\mathrm{P}(T<\infty)=\frac{\phi(b \sqrt{d})}{\phi(|b| \sqrt{d})} .
$$

If $b \geq 0$ then

$$
\mathrm{P}(T<\infty)=1
$$

If $b<0$ then

$$
\mathrm{P}(T<\infty)=\frac{\phi(b \sqrt{d})}{\phi(-b \sqrt{d})}<1 .
$$

We summarize the results in the following theorem.

Theorem C.4. Let $X_{t}=b t+Z_{t}$, where $b$ is some fixed real number, and construct the stopping time $H_{(0,0), d}^{+}(X)$ according to (A.4). Let $\phi$ be the moment generating function defined in (A.2). Then,

$$
\mathrm{P}\left(H_{(0,0), d}^{+}(X)<\infty\right)=\frac{\phi(b \sqrt{d})}{\phi(|b| \sqrt{d})} .
$$

Remark. In the real option context this theorem has the following implications. If $b \geq 0$ then the investment process will take place with probability 1 , while there is a positive probability that the disinvestment process will not take place. If $b<0$ then there is a positive probability that the investment process will not take place. Furthermore, if the investment took place then the disinvestment process would take place with probability 1 .

The next result is a direct consequence of Lemma C. 1 and proves an independence relationship for the process $V_{t}$, starting from $h_{\mathrm{I}}$. In order to emphasize that the starting point for our process is $h_{\mathrm{I}}$, we use the notation $V_{t}^{h_{\mathrm{I}}}$ in the proof of the next lemma.

Lemma C.2. Let $T=H_{\left(h_{\mathrm{I}}, h_{\mathrm{I}}\right), d_{\mathrm{I}}}^{+}(V)$ be the stopping time defined in (A.4), and let $b$ and $X_{t}$ be as defined in (3.1). The following conclusions hold.

(a) The random variables $T \mathbf{1}_{\{T<\infty\}}$ and $V_{T} \mathbf{1}_{\{T<\infty\}}$ are independent under the $\mathrm{P}$-measure if and only if $b \geq 0$. In particular, $T \mathbf{1}_{\{T<\infty\}}$ and $X_{T} \mathbf{1}_{\{T<\infty\}}$ are independent under the P-measure if and only if $b \geq 0$.

(b) The random variables $T \mathbf{1}_{\{T<\infty\}}$ and $V_{T} \mathbf{1}_{\{T<\infty\}}$ are independent under the $\mathrm{P}^{*}$-measure for any $b \in \mathbb{R}$. In particular, $T \mathbf{1}_{\{T<\infty\}}$ and $X_{T} \mathbf{1}_{\{T<\infty\}}$ are independent under the $\mathrm{P}^{*}$-measure for any $b \in \mathbb{R}$.

Proof. We know that $V_{t}$ is the geometric Brownian motion in (2.1). If it starts from $h_{\mathrm{I}}$ then

$$
V_{t}^{h_{\mathrm{I}}}=h_{\mathrm{I}} \exp \left\{\sigma X_{t}\right\}, \quad \text { where } \quad X_{t}=b t+Z_{t} \text { and } b=\frac{\mu-\sigma^{2} / 2}{\sigma} .
$$


From the equality above we also obtain $H_{\left(h_{\mathrm{I}}, h_{\mathrm{I}}\right), d_{\mathrm{I}}}^{+}(V)=H_{(0,0), d_{\mathrm{I}}}^{+}(X)$. Thus, we have obtained

$$
V_{H_{\left(h_{\mathrm{I}}, h_{\mathrm{I}}\right), d_{\mathrm{I}}}^{+}(V)}=h_{\mathrm{I}} \exp \left\{\sigma X_{H_{(0,0), d_{\mathrm{I}}}^{+}(X)}\right\} \quad \text { and } \quad H_{\left(h_{\mathrm{I}}, h_{\mathrm{I}}\right), d_{\mathrm{I}}}^{+}(V)=H_{(0,0), d_{\mathrm{I}}}^{+}(X) .
$$

The independence now follows from Lemma C.1.

We now prove that the Parisian investment time and the position of the underlying value process at that time are independent, which is a remarkable finding of the paper.

Theorem C.5. Assume that $V_{0} \leq h_{\mathrm{I}}$, let $b$ and $X_{t}$ be as defined in (3.1), and recall that the Parisian investment time is $\tau_{\mathrm{I}}=H_{\left(V_{0}, h_{\mathrm{I}}\right), d_{\mathrm{I}}}^{+}(V)$. Then the following conclusions hold.

(a) If $b \geq 0$ then the stopping time $\tau_{\mathrm{I}}$ is finite $\mathrm{P}$-a.s. and, under $\mathrm{P}^{*}, \tau_{\mathrm{I}}$ is finite a.s. for any $b \in \mathbb{R}$.

(b) The random variables $\tau_{\mathrm{I}} \mathbf{1}_{\left\{\tau_{\mathrm{I}}<\infty\right\}}$ and $V_{\tau_{\mathrm{I}}} \mathbf{1}_{\left\{\tau_{\mathrm{I}}<\infty\right\}}$ are independent under the P-measure if and only if $b \geq 0$. In particular, $\tau_{\mathrm{I}} \mathbf{1}_{\left\{\tau_{\mathrm{I}}<\infty\right\}}$ and $X_{\tau_{\mathrm{I}}} \mathbf{1}_{\left\{\tau_{\mathrm{I}}<\infty\right\}}$ are independent under the $\mathrm{P}$-measure if and only if $b \geq 0$.

(c) The random variables $\tau_{\mathrm{I}} \mathbf{1}_{\left\{\tau_{\mathrm{I}}<\infty\right\}}$ and $V_{\tau_{\mathrm{I}}} \mathbf{1}_{\left\{\tau_{\mathrm{I}}<\infty\right\}}$ are independent under the $\mathrm{P}^{*}$-measure for any $b \in \mathbb{R}$. In particular, $\tau_{\mathrm{I}} \mathbf{1}_{\left\{\tau_{\mathrm{I}}<\infty\right\}}$ and $X_{\tau_{\mathrm{I}}} \mathbf{1}_{\left\{\tau_{\mathrm{I}}<\infty\right\}}$ are independent under the $\mathrm{P}^{*}$-measure for any $b \in \mathbb{R}$.

Proof. Let us first prove the independence under P*. We apply Proposition C. 1 with $\tau=0$, and obtain

$$
\tau_{\mathrm{I}}=H_{\left(V_{0}, h_{\mathrm{I}}\right), d_{\mathrm{I}}}^{+}(V)=T_{\left(V_{0}, h_{\mathrm{I}}\right)}(V)+H_{\left(h_{\mathrm{I}}, h_{\mathrm{I}}\right), d_{\mathrm{I}}}^{+}(V),
$$

where the terms of the sum are independent. If $b \geq 0$ then, under $\mathrm{P}, \tau_{\mathrm{I}}<\infty$ a.s. because the stopping times $T_{\left(V_{0}, h_{\mathrm{I}}\right)}(V)$ and $H_{\left(h_{\mathrm{I}}, h_{\mathrm{I}}\right), d_{\mathrm{I}}}^{+}(V)$ are finite. Similarly, $\tau_{\mathrm{I}}<\infty$ a.s. under $\mathrm{P}^{*}$ for any $b \in \mathbb{R}$. On the other hand, by the strong Markov property and the continuity of the process $V$, we have the following equality in distribution under $\mathrm{P}^{*}$ :

$$
V_{\tau_{\mathrm{I}}} \mathbf{1}_{\left\{\tau_{\mathrm{I}}<\infty\right\}}=V_{H_{\left(h_{\mathrm{I}}, h_{\mathrm{I}}\right), d_{\mathrm{I}}}^{+}(V)} .
$$

We now apply Lemma C.2, and find that $V_{H_{\left(h_{\mathrm{I}}, h_{\mathrm{I}}\right), d_{\mathrm{I}}}^{+}(V)}$ and $H_{\left(h_{\mathrm{I}}, h_{\mathrm{I}}\right), d_{\mathrm{I}}}^{+}(V)$ are independent. By the strong Markov property we find that $V_{H_{\left(h_{\mathrm{I}}, h_{\mathrm{I}}\right), d_{\mathrm{I}}}^{+}(V)}$ and $T_{\left(V_{0}, h_{\mathrm{I}}\right)}(V)$ are independent. Therefore, we find that $\tau_{\mathrm{I}}$ and $V_{\tau_{\mathrm{I}}}$ are independent, which is the desired result. The proof of independence under $\mathrm{P}$ is similar to the proof of Lemma C. 1 and so we omit it.

We now have all the 'components' to prove Theorem C.1.

Proof of Theorem C.1. Here we work under the measure $\mathrm{P}^{*}$. Let $\tau_{\mathrm{D}}=H_{\left(V_{0}, h_{\mathrm{D}}\right), d_{\mathrm{D}}}^{-, \tau_{\mathrm{D}}}(V)$. We need to prove that $\tau_{\mathrm{D}}$ and $V_{\tau_{\mathrm{D}}}$ are independent. Recall that the Parisian investment time is $\tau_{\mathrm{I}}=H_{\left(V_{0}, h_{\mathrm{I}}\right), d_{\mathrm{I}}}^{+}(V)$ and that, by Theorem C.5, the random variables $\tau_{\mathrm{I}}$ and $V_{\tau_{\mathrm{I}}}$ are independent. We apply Proposition C.1 with $\tau=\tau_{\mathrm{I}}$ and obtain

$$
\tau_{\mathrm{D}}=H_{\left(V_{0}, h_{\mathrm{D}}\right), d_{\mathrm{D}}}^{-, \tau_{\mathrm{I}}}(V)=\tau_{\mathrm{I}}+T_{V_{\tau_{\mathrm{I}}}, h_{\mathrm{D}}}(V)+H_{\left(h_{\mathrm{D}}, h_{\mathrm{D}}\right), d_{\mathrm{D}}}^{-}(V),
$$

where the terms of the sum are independent. Also, under $\mathrm{P}^{*}, \tau_{\mathrm{D}}<\infty$ because the stopping times $\tau_{\mathrm{I}}, T_{V_{\tau_{\mathrm{I}}}, h_{\mathrm{D}}}(V)$, and $H_{\left(h_{\mathrm{D}}, h_{\mathrm{D}}\right), d_{\mathrm{D}}}^{-}(V)$ are finite. On the other hand, by the strong Markov property and the continuity of the process $V$, we have the following equality in distribution under $\mathrm{P}^{*}$ :

$$
V_{\tau_{\mathrm{D}}}=V_{H_{\left(h_{\mathrm{D}}, h_{\mathrm{D}}\right), d_{\mathrm{D}}}^{-}}(V)
$$


We now apply Lemma C.2, and find that $V_{H_{\left(h_{\mathrm{D}}, h_{\mathrm{D}}\right), d_{\mathrm{D}}}^{-}}(V)$ and $H_{\left(h_{\mathrm{D}}, h_{\mathrm{D}}\right), d_{\mathrm{D}}}^{-}(V)$ are independent. By the strong Markov property, we find that $V_{H_{\left(h_{\mathrm{D}}, h_{\mathrm{D}}\right), d_{\mathrm{D}}}^{\left(h_{\mathrm{D}}, h_{\mathrm{D}}\right), d_{\mathrm{D}}}(V)}(V)$ independent of $\tau_{\mathrm{I}}$ and of $T_{V_{\tau_{\mathrm{I}}}, h_{\mathrm{D}}}(V)$. Therefore, we find that $\tau_{\mathrm{I}}$ and $V_{\tau_{\mathrm{I}}}$ are independent, which is the desired result.

\section{Appendix D. Laplace transforms and moment generating functions}

To obtain the optimal investment and disinvestment thresholds in analytic form, we need to calculate all terms that enter into the maximization problem. We first find the Laplace transform of the Parisian investment time under the measure $\mathrm{P}^{*}$ defined in (3.2).

Proposition D.1. For any $\lambda>0$, the following equality holds:

$$
\mathrm{E}_{\mathrm{P}^{*}}\left[\exp \left\{-\lambda \tau_{\mathrm{I}}\right\}\right]=\left(\frac{V_{0}}{h_{\mathrm{I}}}\right)^{\sqrt{2 \lambda} / \sigma} \frac{1}{\phi\left(\sqrt{2 \lambda d_{\mathrm{I}}}\right)} .
$$

Proof. From Proposition C.1 we have

$$
\mathrm{E}_{\mathrm{P}^{*}}\left[\exp \left\{-\lambda \tau_{\mathrm{I}}\right\}\right]=\mathrm{E}_{\mathrm{P}^{*}}\left[\exp \left\{-\lambda T_{l_{0}, l_{\mathrm{I}}}\right\}\right] \mathrm{E}_{\mathrm{P}}\left[\exp \left\{-\lambda H_{\left(l_{\mathrm{I}}, l_{\mathrm{I}}\right), d_{\mathrm{I}}}^{+}(X)\right\}\right] .
$$

Using the corresponding Laplace transforms, we obtain

$$
\mathrm{E}_{\mathrm{P} *}\left[\exp \left\{-\lambda \tau_{\mathrm{I}}\right\}\right]=\exp \left\{-\left(l_{\mathrm{I}}-l_{0}\right) \sqrt{2 \lambda}\right\} \frac{1}{\phi\left(\sqrt{2 \lambda d_{\mathrm{I}}}\right)}=\left(\frac{V_{0}}{h_{\mathrm{I}}}\right)^{\sqrt{2 \lambda} / \sigma} \frac{1}{\phi\left(\sqrt{2 \lambda d_{\mathrm{I}}}\right)} .
$$

In the next proposition we calculate the moment generating function for the process $X_{t}$ defined in (3.1), stopped at the Parisian investment time.

Proposition D.2. For any $\lambda \in \mathbb{R}$, the following equality holds:

$$
\mathrm{E}_{\mathrm{P} *}\left[\exp \left\{-\lambda X_{\tau_{\mathrm{I}}}\right\}\right]=\left(\frac{h_{\mathrm{I}}}{V_{0}}\right)^{-\lambda / \sigma} \phi\left(-\lambda \sqrt{d_{\mathrm{I}}}\right) .
$$

Proof. Using the definition of $X_{\tau_{\mathrm{I}}}$,

$$
\mathrm{E}_{\mathrm{P} *}\left[\exp \left\{-\lambda X_{\tau_{\mathrm{I}}}\right\}\right]=\mathrm{E}_{\mathrm{P}}\left[\exp \left\{-\lambda\left(l_{\mathrm{I}}+m_{1} \sqrt{d_{\mathrm{I}}}\right)\right\}\right]
$$

Now, using the definitions of $l_{\mathrm{I}}$ and $\phi$, we obtain

$$
\mathrm{E}_{\mathrm{P} *}\left[\exp \left\{-\lambda X_{\tau_{\mathrm{I}}}\right\}\right]=\exp \left\{-\lambda l_{\mathrm{I}}\right\} \phi\left(-\lambda \sqrt{d_{\mathrm{I}}}\right)=\left(\frac{h_{\mathrm{I}}}{V_{0}}\right)^{-\lambda / \sigma} \phi\left(-\lambda \sqrt{d_{\mathrm{I}}}\right) .
$$

In the following we calculate the Laplace transform of the first hitting time of $X$, starting at the Parisian investment time.

Proposition D.3. For any $\lambda>0$, the following equality holds:

$$
\mathrm{E}_{\mathrm{P}}\left[\exp \left\{-\lambda T_{\left(X_{\tau_{\mathrm{I}}}, l_{\mathrm{D}}\right)}(X)\right\}\right]=\left(\frac{h_{\mathrm{D}}}{h_{\mathrm{I}}}\right)^{\sqrt{2 \lambda} / \sigma} \phi\left(-\sqrt{2 \lambda d_{\mathrm{I}}}\right) .
$$


Proof. Conditioning, we write

$$
\mathrm{E}_{\mathrm{P} *}\left[\exp \left\{-\lambda T_{\left(X_{\tau_{\mathrm{I}}}, l_{\mathrm{D}}\right)}(X)\right\}\right]=\mathrm{E}_{\mathrm{P}}\left[\mathrm{E}_{\mathrm{P}}\left[\exp \left\{-\lambda T_{\left(X_{\tau_{\mathrm{I}}}, l_{\mathrm{D}}\right)}(X)\right\} \mid \mathcal{F}_{\tau_{\mathrm{I}}}\right]\right] .
$$

Since $X_{\tau_{\mathrm{I}}} \geq l_{\mathrm{D}}$ a.s., we can use the Laplace transform of the hitting time to obtain

$$
\mathrm{E}_{\mathrm{P} *}\left[\exp \left\{-\left(X_{\tau_{\mathrm{I}}}-l_{\mathrm{D}}\right) \sqrt{2 \lambda}\right\}\right]
$$

Using the formulae for $X_{\tau_{\mathrm{I}}}$ and $l_{d}$, we know that $X_{\tau_{\mathrm{I}}}-l_{d}=(1 / \sigma) \log \left(V_{\tau_{\mathrm{I}}} / h_{\mathrm{D}}\right)$ and, hence, we obtain

$$
\mathrm{E}_{\mathrm{P}}\left[\left(\frac{V_{\tau_{\mathrm{I}}}}{h_{\mathrm{D}}}\right)^{-\sqrt{2 \lambda} / \sigma}\right]=h_{\mathrm{D}}^{\sqrt{2 \lambda} / \sigma} \mathrm{E}_{\mathrm{P} *}\left[V_{\tau_{\mathrm{I}}}^{-\sqrt{2 \lambda} / \sigma}\right]=\left(\frac{h_{\mathrm{D}}}{V_{0}}\right)^{\sqrt{2 \lambda} / \sigma} \mathrm{E}_{\mathrm{P}^{*}}\left[\exp \left\{-\sqrt{2 \lambda} X_{\tau_{\mathrm{I}}}\right\}\right] .
$$

Applying Proposition D.2 we obtain the desired result.

Now, we find the Laplace transform of the Parisian disinvestment time under the measure $\mathrm{P}^{*}$ defined in (3.2).

Proposition D.4. For any $\lambda>0$, the following equality holds:

$$
\mathrm{E}_{\mathrm{P} *}\left[\exp \left\{-\lambda \tau_{\mathrm{D}}\right\}\right]=\mathrm{E}_{\mathrm{P} *}\left[\exp \left\{-\lambda \tau_{\mathrm{I}}\right\}\right] \frac{\phi\left(-\sqrt{2 \lambda d_{\mathrm{I}}}\right)}{\phi\left(\sqrt{2 \lambda d_{\mathrm{D}}}\right)}\left(\frac{h_{\mathrm{D}}}{h_{\mathrm{I}}}\right)^{\sqrt{2 \lambda} / \sigma} .
$$

Proof. Using Proposition C.1, we can write

$$
\mathrm{E}_{\mathrm{P}^{*}}\left[\exp \left\{-\lambda \tau_{\mathrm{D}}\right\}\right]=\mathrm{E}_{\mathrm{P}^{*}}\left[\exp \left\{-\lambda \tau_{\mathrm{I}}\right\}\right] \mathrm{E}_{\mathrm{P}^{*}}\left[\exp \left\{-\lambda T_{\left(X_{\tau_{\mathrm{I}}}, l_{\mathrm{D}}\right)}(X)\right\}\right] \mathrm{E}_{\mathrm{P}}\left[\exp \left\{-\lambda H_{\left(l_{\mathrm{D}}, l_{\mathrm{D}}\right), d_{\mathrm{D}}}^{-}(X)\right\}\right] .
$$

Now, using the corresponding Laplace transforms, we obtain the desired result.

Again, we calculate the moment generating function for the process $X_{t}$ defined in (3.1), stopped at the Parisian disinvestment time.

Proposition D.5. For any $\lambda \in \mathbb{R}$, the following equality holds:

$$
\mathrm{E}_{\mathrm{P}^{*}}\left[\exp \left\{-\lambda X_{\tau_{\mathrm{D}}}\right\}\right]=\left(\frac{h_{\mathrm{D}}}{V_{0}}\right)^{-\lambda / \sigma} \phi\left(\lambda \sqrt{d_{\mathrm{D}}}\right) .
$$

Proof. Using the definition of $X_{\tau_{\mathrm{D}}}$,

$$
\mathrm{E}_{\mathrm{P} *}\left[\exp \left\{-\lambda X_{\tau_{\mathrm{D}}}\right\}\right]=\mathrm{E}_{\mathrm{P}}\left[\exp \left\{-\lambda\left(l_{\mathrm{D}}-m_{1} \sqrt{d_{\mathrm{D}}}\right)\right\}\right] .
$$

Now, using the definitions of $l_{\mathrm{D}}$ and $\phi$, we obtain the desired result.

Finally, we are able to calculate the first term appearing in the maximization problem, (2.2).

Proposition D.6. The following equality holds:

$$
\begin{aligned}
\mathrm{E}_{\mathrm{P}}\left[\exp \left\{-\rho \tau_{\mathrm{I}}\right\}\left(V_{\tau_{\mathrm{I}}}-C_{\mathrm{I}}\right) \mathbf{1}_{\left\{\tau_{\mathrm{I}}<\infty\right\}}\right]= & \mathrm{E}_{\mathrm{P}^{*}}\left[\exp \left\{-\left(\rho+\frac{b^{2}}{2}\right) \tau_{\mathrm{I}}\right\}\right]\left(\frac{h_{\mathrm{I}}}{V_{0}}\right)^{b / \sigma} \phi\left(b \sqrt{d_{\mathrm{I}}}\right) \\
& \times\left(h_{\mathrm{I}} \frac{\phi\left(\sqrt{d_{\mathrm{I}}}(\sigma+b)\right)}{\phi\left(b \sqrt{d_{\mathrm{I}}}\right)}-C_{\mathrm{I}}\right) .
\end{aligned}
$$


Proof. Using (3.3) and Proposition D.2, the left-hand side of the above equality becomes

$$
\mathrm{E}_{\mathrm{P}^{*}}\left[\exp \left\{-\left(\rho+\frac{b^{2}}{2}\right) \tau_{\mathrm{I}}\right\}\right]\left(V_{0}\left(\frac{h_{\mathrm{I}}}{V_{0}}\right)^{(\sigma+b) / \sigma} \phi\left(\sqrt{d_{\mathrm{I}}}(\sigma+b)\right)-C_{\mathrm{I}}\left(\frac{h_{\mathrm{I}}}{V_{0}}\right)^{b / \sigma} \phi\left(b \sqrt{d_{\mathrm{I}}}\right)\right)
$$

and grouping the terms we obtain the desired result.

Similarly, we calculate the second term appearing in the maximization problem, (2.2).

Proposition D.7. The following equality holds:

$$
\begin{aligned}
\mathrm{E}_{\mathrm{P}}\left[\exp \left\{-\rho \tau_{\mathrm{D}}\right\}\left(C_{\mathrm{D}}-V_{\tau_{\mathrm{D}}}\right) \mathbf{1}_{\left\{\tau_{\mathrm{D}}<\infty\right\}}\right] \\
=\mathrm{E}_{\mathrm{P}^{*}}\left[\exp \left\{-\left(\rho+\frac{b^{2}}{2}\right) \tau_{\mathrm{I}}\right\}\right]\left(\frac{h_{\mathrm{I}}}{V_{0}}\right)^{b / \sigma}\left(\frac{h_{\mathrm{I}}}{h_{\mathrm{D}}}\right)^{\left(-b-\sqrt{2 \rho+b^{2}}\right) / \sigma} \\
\quad \times \frac{\phi\left(-\sqrt{\left(2 \rho+b^{2}\right) d_{\mathrm{I}}}\right)}{\phi\left(\sqrt{\left(2 \rho+b^{2}\right) d_{\mathrm{D}}}\right)} \phi\left(-b \sqrt{d_{\mathrm{D}}}\right)\left(C_{\mathrm{D}}-h_{\mathrm{D}} \frac{\phi\left(-(b+\sigma) \sqrt{d_{\mathrm{D}}}\right)}{\phi\left(-b \sqrt{d_{\mathrm{D}}}\right)}\right) .
\end{aligned}
$$

Proof. Using (3.4), Proposition D.4, and Proposition D.5, the left-hand side of the above equality becomes

$$
\begin{aligned}
& \mathrm{E}_{\mathrm{P}^{*}}\left[\exp \left\{-\left(\rho+\frac{b^{2}}{2}\right) \tau_{\mathrm{I}}\right\}\right]\left(\frac{h_{\mathrm{D}}}{h_{\mathrm{I}}}\right)^{\sqrt{2 \rho+b^{2}} / \sigma} \frac{\phi\left(-\sqrt{\left(2 \rho+b^{2}\right) d_{\mathrm{I}}}\right)}{\phi\left(\sqrt{\left(2 \rho+b^{2}\right) d_{\mathrm{D}}}\right)} \\
& \times\left(C_{\mathrm{D}}\left(\frac{h_{\mathrm{D}}}{V_{0}}\right)^{b / \sigma} \phi\left(-b \sqrt{d_{\mathrm{D}}}\right)-V_{0}\left(\frac{h_{\mathrm{D}}}{V_{0}}\right)^{(\sigma+b) / \sigma} \phi\left(-(b+\sigma) \sqrt{d_{\mathrm{D}}}\right)\right),
\end{aligned}
$$

and now factoring out and grouping the terms we obtain the desired result.

\section{Acknowledgements}

Part of Taschini's research was supported by the University Research Priority Program 'Finance and Financial Markets' and by the National Centre of Competence in Research 'Financial Valuation and Risk Management' (NCCR FINRISK), which are respectively research instruments of the University of Zürich and of the Swiss National Science Foundation. The authors would like to thank Marc Chesney, Kais Hamza, and Mihail Zervos for their helpful discussions and comments.

\section{References}

Avellaneda, M. and Wu, L. (1999). Pricing Parisian-style options with a lattice method. Internat. J. Theoret. Appl. Finance 2, 1-16.

Bar-Ilan, A. And Strange, C. (1996). Investment lags. Amer. Econom. Rev. 86, 610-622.

Black, F. ANd Scholes, M. S. (1973). The pricing of options and corporate liabilities. J. Political Econom. 7, $637-654$.

BreKKe, K. A. AND ØKSEndAL, B. (1994). Optimal switching in an economic activity under uncertainty. SIAM J. Control Optimization 32, 1021-1036.

Brennan, M. J. ANd Schwartz, E. S. (1985). Evaluating natural resource investments. J. Business 58, $135-157$.

Chesney, M., Jeanblanc, M. And Yor, M. (1997). Brownian excursions and Parisian barrier options. Adv. Appl. Prob. 29, 165-184.

DENG, S. AND OREN, S. S. (2003). Incorporating operational characteristics and start-up costs in option-based valuation of power generation capacity. Prob. Eng. Inf. Sci. 17, 155-181.

Dixit, A. (1989). Entry and exit decisions under uncertainty. J. Political Econom. 97, 620-638.

Dixit, A. K. AND PINDYCK, R. S. (1994). Investment Under Uncertainty. Princeton University Press. 
Duckworth, K. J. and Zervos, M. (2000). An investment model with entry and exit decisions. J. Appl. Prob. 37, 547-559.

Gauthier, L. And Morellec, E. (2000). Investment under uncertainty with implementation delay. In New Developments and Applications in Real Options. Oxford University Press.

Haber, R. J., Schonbucker, P. J. and Wilmott, P. (1999). Pricing Parisian options. J. Derivatives 6, 71-79.

Hamadène, S. AND JEAnblanc, M. (2007). On the starting and stopping problem: application in reversible investments. Math. Operat. Res. 32, 182-192.

Harris, M. AND Raviv, A. (1996). The capital budgeting process: incentives and information. J. Finance 51, 1139-1174.

Knudsen, T. S., Meister, B. And Zervos, M. (1999). On the relationship of the dynamic programming approach and the contingent claim approach to asset valuation. Finance Stoch. 3, 433-449.

MaJd, S. AND PINDYCK, R. S. (1987). Time to build, option value, and investment decisions. J. Financial Econom. 18, 7-27.

McDonald, R. And Siegel, D. (1986). The value of waiting to invest. Quart. J. Econom. 101, 707-728.

Merton, R. C. (1973). Theory of rational option pricing. Bell J. Econom. 4, 141-183.

ØKSENDAL, B. (2005). Optimal stopping with delayed information. Stoch. Dyn. 5, 271-280.

PINDYCK, R. S. (1991). Irreversibility, uncertainty, and investment. J. Econom. Literature 29, 1110-1148.

PINDYCK, R. S. (1993). Investments of uncertain cost. J. Financial Econom. 34, 53-76.

Revuz, D. AND Yor, M. (1991). Continuous Martingales and Brownian Motion. Springer, Berlin.

SCHRöDER, M. (2003). Brownian excursions and Parisian barrier options: a note. J. Appl. Prob. 40, 855-864.

Tseng, C.-L. AND BARz, G. (2002). Short-term generation asset valuation: a real options approach. Operat. Res. 50, 297-310. 